\title{
LA FUNCIÓN SOCIAL DE LAS COOPERATIVAS: NOTAS DE DERECHO COMPARADO
}

\author{
POR \\ Antonio FICI ${ }^{1}$
}

\section{RESUMEN}

Muchas Constituciones en el mundo atribuyen una función social a las sociedades cooperativas y prevén que el legislador las promueva. La ley y las instituciones europeas suelen incluir a las cooperativas en las entidades de la economía social que los poderes públicos tienen la tarea de fomentar. Para que estas normas puedan considerarse legítimas a la luz del principio constitucional de igualdad ante la ley, las cooperativas deberán poseer una identidad jurídica distinta de la de otras sociedades. Este artículo se propone identificar los principales elementos de la identidad jurídica de la cooperativa que la hacen un tipo de sociedad digna de específica tutela y trato privilegiado en la Constitución y por parte de la ley. Además, el artículo tratará de la distinción entre cooperativas mutualistas y cooperativas de interés general a la luz del problema de la regulación de la "empresa social".

Palabras clave: Cooperativas, función social, fin de mutualidad, interés general, empresa social.

Claves Econlit: M140

\section{THE SOCIAL FUNCTION OF COOPERATIVES: COMPARATIVE LAW NOTES}

\begin{abstract}
Many Constitutions around the world attribute a social function to cooperatives and provide for their promotion by the law. The law and the European institutions include cooperatives among the entities of the social economy, which the State has the duty to
\end{abstract}

\footnotetext{
${ }^{1}$ Profesor de Derecho comparado de las sociedades cooperativas. Departimento jurídico. LUMSA de Roma. Dirección de correo electrónico: antonio.fici@ unimol.it. REVESCO $\mathrm{N}^{\circ} 117$ - MONOGRÁFICO: Las sociedades cooperativas construyen un mundo mejor - ISSN: 1885-8031 - www.ucm.es/info/revesco

http://dx.doi.org/10.5209/rev_REVE.2015.v117.48146

Fecha de recepción: 02/12/2014

Fecha de aceptación: 21/01/2015
} 
support. For these provisions to be legitimate with regard to the constitutional principle of equal treatment by law, cooperatives must have a legal identity different from that of other business organizations. This article aims at identifying the principal elements of the legal identity of a cooperative, which make it a type of society worth of a specific protection and privileged treatment in the Constitution and the law. The article will also discuss the distinction between mutual cooperatives and general interest cooperatives, also in light of the issue of the "social enterprise" regulation.

Keywords: Cooperatives, social function, mutual purpose, general interest, social enterprise

\section{INTRODUCIÓN. LA LEY Y LA FUNCIÓN SOCIAL DE LAS COOPERATIVAS}

Muchas Constituciones en el mundo atribuyen una función social a las sociedades cooperativas y prevén que el legislador las promueva, lo que en cambio no reconocen a otros tipos de sociedades.

Limitándonos a Europa, esto es lo que ocurre, por ejemplo, en España, Italia y, a través de una enorme cantidad de disposiciones, en Portugal ${ }^{2}$.

Desde esta misma perspectiva, la ley y las instituciones europeas suelen incluir a las cooperativas en las entidades de la economía social que los poderes públicos tienen la tarea de fomentar ${ }^{3}$.

Para merecer estas específicas disposiciones a su favor, o en otras palabras, para que estas normas puedan considerarse legítimas a la luz del principio constitucional de igualdad

\footnotetext{
${ }^{2}$ El art. 45 de la Constitución italiana de 1948 establece que "La República reconoce la función social de la cooperación con carácter de mutualidad y sin fines de especulación privada. La ley promueve y favorece el incremento de la misma con los medios más adecuados y asegura, a través de los controles oportunos, su carácter y sus finalidades".

Según el art. 129, apartado 2, de la Constitución española de 1978, "Los poderes públicos promoverán eficazmente las diversas formas de participación en la empresa y fomentarán, mediante una legislación adecuada, las sociedades cooperativas. También establecerán los medios que faciliten el acceso de los trabajadores a la propiedad de los medios de producción”.

Con respecto a la Constitución portuguesa de 1976, véanse los artículos 43, apartado 4; 60, apartado 3; 61 apartados 2 y 3; 65, apartado 2, d); 80 b) y f); 82, apartado 4; 85; 94, apartado 2; 97, apartado 1 y 2, d); 136, apartado 3, b); 288, f).

Como justamente recuerdan (Gadea, Sacristán y Vargas Vasserot, 2009: 37), estos preceptos constituyen un mandato dirigido a los poderes públicos para que éstos fomenten las sociedades cooperativas.

${ }^{3}$ Véanse, entre otras, art. 5 de la Ley n ${ }^{\circ}$ 5/2011 de 29 de marzo 2011, de economía social, en España; art. 4 de la Ley $n^{\circ}$ 30/2013 de 8 de mayo 2013, de bases de economía social, en Portugal: sobre la cual véase (Aparício Meira, 2013: 21 y ss.); y ahora también art. 1 de la Ley n ${ }^{\circ}$ 387/2014 de 21 de julio 2014, de economía social y solidaria, en Francia; véase también Comisión Europea, COM(2011) 682 final, de 25.10.2011, Iniciativa en favor del emprendimiento social, p. 3.
}

REVESCO No 117 - MONOGRÁFICO: Las sociedades cooperativas construyen un mundo mejor - ISSN: 1885-8031 - www.ucm.es/info/revesco 
ante la ley ${ }^{4}$, las cooperativas deberán presentar rasgos particulares que las destaquen entre los varios tipos de sociedades, es decir, deberán poseer una identidad jurídica distinta de la de otras sociedades.

Este artículo se propone identificar los principales elementos de la identidad jurídica de la cooperativa que la hacen un tipo de sociedad digna de específica tutela y trato privilegiado en la Constitución y por parte de la ley.

A este respecto, y antes de entrar en medias res, dos puntualizaciones parecen oportunas.

En primer lugar, el análisis que se desarrollará en este artículo no podrá sino centrarse en el régimen jurídico de las cooperativas y en su identidad jurídica. De hecho, las cooperativas constituyen un tipo de sociedad entre otros tipos previstos por el legislador. Por consiguiente, el legislador no podría reconocer a las cooperativas una función social y un trato particular en virtud de rasgos distintivos que él se abstenga de definir (de forma directa o también indirecta, por ejemplo haciendo referencia a los principios de la Alianza Cooperativa Internacional $-\mathrm{ACI}^{5}$ ).

En este sentido, el Derecho conforma las cooperativas y su identidad, así que, si se quiere comprender porque las cooperativas tienen una función social reconocida por la ley, se debe ir en la búsqueda de los aspectos del régimen jurídico de las cooperativas que delinean esta función social.

Aquí el razonamiento se vuelve un poco circular. En la medida en que la ley reconoce la función social de las cooperativas, y sin embargo esta función social es un producto de la ley, el régimen jurídico de las cooperativas tendrá que contemplar normas apropiadas para que se pueda decir que realmente las cooperativas realizan una función social. Destacar estas normas forma parte de los objetivos de este artículo.

\footnotetext{
${ }^{4}$ Véanse los artículos 3 de la Constitución italiana, 14 de la Constitución española, y 13 de la Constitución portuguesa.

${ }_{5}^{5}$ Muchas leyes cooperativas en el mundo contienen una referencia expresa a los principios de la ACI (véase, entre otros, art. 1, apartado 1, de la Ley estatal española $n^{\circ}$ 27/1999) o incluso reproducen literalmente estos principios (véase, entre otros, el art. 3, del Código cooperativo portugués de 1996). La Declaración de identidad cooperativa (en su tercera versión de 1995) en la que estos principios están contenidos puede leerse en http://www.aciamericas.coop/Principios-y-Valores-Cooperativos-4456.
}

REVESCO No 117 - MONOGRÁFICO: Las sociedades cooperativas construyen un mundo mejor - ISSN: 1885-8031 - www.ucm.es/info/revesco 
En segundo lugar, una vez que la ley haya decidido instituir un tipo específico de sociedad con función social cual es la cooperativa, el trato diferente que la misma ley decida acordar para este tipo societario, por ejemplo en el Derecho tributario, de la competencia o de la contratación pública, no sería un trato privilegiado en sentido estricto, sino un trato específico como el tipo societario al que se aplica. Por ello, identificar la especificidad del régimen sustancial de las cooperativas frente al de otros tipos de sociedades deviene central para justificar su régimen específico de Derecho tributario o en otras áreas del Derecho. A la inversa, y aquí de nuevo el discurso se hace circular, para brindar a las cooperativas un trato más favorable que a las otras sociedades en el Derecho tributario u otras áreas del Derecho, es necesario proporcionar a las mismas una identidad específica y distinta en el Derecho sustancial.

Que esto sea fundamental también y sobre todo a nivel supranacional, lo demuestra la sentencia del Tribunal de Justicia de la Unión Europa (TJUE) de 8 de septiembre de $2011^{6}$. En esta decisión, una ley sustancial, como el Reglamento de la Sociedad Cooperativa Europea (SCE) de 2003, asumió un papel decisivo para que el Tribunal declarara potencialmente compatible con el Derecho comunitario (más concretamente, con la regulación de ayudas de Estado) una tributación de las cooperativas diferente de (y más favorable que) la de las otras organizaciones empresariales ${ }^{7}$.

El TJUE - si bien reconoce que, por lo general, las exenciones fiscales constituyen una ayuda de Estado - niega, sin embargo, que dichas medidas sean incompatibles con el Derecho de la UE, teniendo en cuenta la diferencia entre las cooperativas y los operadores constituidos como entidades jurídicas con ánimo de lucro, lo que justifica y legitima el tratamiento fiscal distinto y más favorable del que gozan las primeras ${ }^{8}$.

\footnotetext{
${ }^{6}$ Véase Tribunal de Justicia de la Unión Europea, 8 de Septiembre de 2011 (C-78/08 a C-80/08).

${ }^{7}$ La sentencia del TJUE tiene origen en una petición de decisión prejudicial planteada por la Corte Suprema Italiana (Corte Suprema di Cassazione) según lo establecido en el art. 267 del Tratado de Funcionamiento de la Unión Europea (TFUE) (antiguo art. 234 del Tratado constitutivo de la Comunidad Europea - TCE), y relativa a la compatibilidad con el Derecho comunitario de las exenciones fiscales otorgadas por la ley italiana a las sociedades cooperativas de producción y trabajo. Más concretamente, la Corte Suprema Italiana planteó sustancialmente la cuestión acerca de si estas ventajas podrían considerarse ayuda de Estado y, de ser así, en qué medida en la acepción del art. 107 del TFUE (antiguo art. 87 TCE). Las medidas fiscales estaban previstas en el art. 11, Decreto Presidencial $\mathrm{n}^{\circ}$ 601/1973. Sobre las principales medidas fiscales aplicables a las cooperativas italianas, véase (Fici, 2013c: 496 y ss.).

${ }^{8}$ Vid. TJUE, 8 de septiembre 2011, apartado 82 y la resolución definitiva.
}

REVESCO No 117 - MONOGRÁFICO: Las sociedades cooperativas construyen un mundo mejor - ISSN: 1885-8031 - www.ucm.es/info/revesco 
El Tribunal llega a esta conclusión fundamentándose en una elaborada sentencia que no se puede resumir aquí ${ }^{\prime}$. Lo que cabe únicamente subrayar es que el TJUE basa la distinta identidad de las cooperativas en rasgos que extrae del Reglamento de la SCE, es decir, de un texto legal. Por lo tanto, como ya se señalaba antes, la existencia de normas de Derecho sustancial que diferencien las cooperativas de otros tipos de sociedades sirve para justificar un régimen promocional de las cooperativas dispuesto por otras normas (de Derecho de la competencia y de ayuda de Estado).

Todo esto demuestra que, en general, el desarrollo de las cooperativas presupone la ley, y más concretamente una ley que les atribuya una específica identidad jurídica.

\section{EL FIN DE MUTUALIDAD DE LAS COOPERATIVAS ...}

El fin es el principal, aunque no el único, elemento de identificación de un tipo jurídico de organización, así también de clasificación de los diferentes tipos de entes previstos por el legislador en un determinado ordenamiento ${ }^{10}$.

En general, es posible identificar tres fines principales que los entes jurídicos pueden adoptar como propio objetivo final: el fin de lucro, el fin no de lucro, y el fin de mutualidad ${ }^{11}$.

El fin de lucro - que en muchos ordenamientos es el objetivo final que la ley expresamente asigna a las sociedades (no cooperativas) ${ }^{12}$ - implica desarrollar una empresa con la finalidad de obtener beneficios para distribuir posteriormente entre los socios. Por lo

\footnotetext{
${ }^{9}$ Sin embargo, véase (Fici, 2014c).

${ }^{10}$ En la doctrina italiana, véase sobre todo (Spada, 1974: 172 y ss.).

${ }^{11}$ Como todas las taxonomías generales, también la citada en el texto admite formas híbridas de entes, que de hecho se pueden encontrar o como resultado de una provisión de ley o de una autónoma decisión de los entes mismos que no está prohibida por la ley (por ejemplo, una sociedad mercantil cuyo estatuto social prevea la asignación de una parte no irrelevante de los beneficios a medidas de "responsabilidad social empresarial"). Esto, como veremos, podría referirse también a las cooperativas, en la medida en que algunos ordenamientos las separen, totalmente o parcialmente, de la persecución del fin de mutualidad.

12 Véase por ejemplo el art. 2247 del Código civil italiano. Sin embargo, no faltan leyes sobre sociedades mercantiles que no expresan los fines de éstas sino sólo la naturaleza comercial de su actividad, como por ejemplo, a nivel supranacional, el Reglamento sobre la sociedad anónima europea (SE) $\mathrm{n}^{\circ}$ 2157/2001. Es muy significativo el hecho de que haya una tendencia a separar las sociedades mercantiles de la persecución de un fin de lucro, como ocurrió, por ejemplo, en Perú en 1984 cuando fue reformada la Ley de sociedades: véase (Torres Morales, 2013: 588-589).

En segundo lugar, cabe subrayar que los legisladores están promulgando cada vez más leyes que instituyen (y por lo tanto permiten establecer) sociedades mercantiles sin ánimo de lucro, incluso sociedades mercantiles de interés general de la comunidad, como veremos después analizando la legislación en tema de "empresa social" (véase sección 5).

Todo esto parece demostrar la validez de la hipótesis formulada hace más que 40 años por un autor italiano, Gerardo Santini, sobre el declive del fin de lucro en las sociedades da capitales: véase (Santini, 1973).
}

REVESCO No 117 - MONOGRÁFICO: Las sociedades cooperativas construyen un mundo mejor - ISSN: 1885-8031 - www.ucm.es/info/revesco 
tanto, las entidades con finalidad de lucro tienen un objetivo que, en términos jurídicos, es "económico" e "interno/egoísta", en la medida en que éstas están orientadas a sus socios cuya riqueza intentan aumentar o maximizar.

Por el contrario, el fin no lucrativo -que en muchos ordenamientos es el objetivo que la ley expresamente asigna a asociaciones y fundaciones- implica conducir una actividad sin el objetivo de obtener beneficios para distribuir posteriormente entre los socios (y/o fundadores, gerentes, empleados, etc.) ${ }^{13}$. Esto no significa que una entidad sin ánimo de lucro no pueda en principio desarrollar una actividad económica y sacar beneficios de ella, sino sólo que estos eventuales beneficios no deberán ser otorgados a los socios (y/o fundadores, gerentes, empleados, etc.) y deberán ser (re)empleados solamente para la consecución del fin $^{14}$. Sin embargo, en términos positivos, este fin (y la actividad necesaria para lograrlo) podría realizar el interés (también económico, si bien no lucrativo en sentido estricto) de los socios o de terceros no socios, incluso de la comunidad. Por consiguiente, las entidades sin ánimo de lucro pueden tener un objetivo tanto de naturaleza "interna/egoísta" como de naturaleza "externa/altruista", lo que depende de si estas entidades aspiran a beneficiar a sus socios o a no $\operatorname{socios}^{15}$.

\footnotetext{
${ }^{13}$ Este fin, por lo tanto, está identificado en términos puramente negativos y en contraste con el fin de lucro. Sin embargo, cabe subrayar que el fin no lucrativo es más amplio que el fin de lucro, puesto que, como diremos a continuación, el primero es en principio compatible con el desarrollo de una actividad de empresa, lo que significa que la naturaleza de la actividad no es relevante para conceptualizar las entidades no lucrativas.

${ }^{14}$ Por consiguiente, lo que califica las entidades no lucrativas es el "non-distribution constraint" (la limitación en la distribución de los beneficios), y no el hecho de que ellas no puedan obtener beneficios de una actividad económica, como correctamente remarca (Hansmann, 1996: 17 y ss.), según el cual, por lo tanto, los miembros de una entidad lucrativa no pueden considerarse sus "propietarios", dado que les falta el derecho de apropiarse de los beneficios residuales de la empresa, derecho que, junto con el derecho de controlar la empresa, es un elemento esencial del concepto de propiedad de la empresa.

La teórica compatibilidad del fin no lucrativo con el desarrollo de actividad económica no impide que algunos legisladores la prohiban a las entidades no lucrativas o la sometan a determinadas condiciones.

${ }^{15}$ No se debe poner demasiado énfasis en esta dicotomía, puesto que la búsqueda del interés externo o general puede ser compatible con una actividad desarrollada también, aunque no sólo, con los miembros del ente, en la medida en que estos miembros pertenecen a la categoría de los beneficiarios, y satisfaciendo sus intereses la entidad no lucrativa persigue su objetivo altruista o de interés general.

La calificación del fin como "altruista" no implica necesariamente una evaluación positiva de este fin por lo que se refiere al bienestar social o general. "Altruista" aquí es solamente opuesto a "interno". Un fin "altruista" puede surtir o no surtir efectos positivos en la sociedad y la comunidad, lo que depende de muchos factores, particularmente la identidad de los beneficiarios y la naturaleza de los intereses servidos por las entidades no lucrativas. Por ello, las entidades no lucrativas no pueden automáticamente considerarse entidades sociales o de interés general, mientras que esta última calificación podría otorgarse a entidades, como las cooperativas, que tienen una función social no obstante sean caracterizadas por un fin no "altruista" en sentido estricto.
}

REVESCO No 117 - MONOGRÁFICO: Las sociedades cooperativas construyen un mundo mejor - ISSN: 1885-8031 - www.ucm.es/info/revesco 
El fin de mutualidad es el que caracteriza a las cooperativas ${ }^{16}$. Debe ser distinguido tanto del fin lucrativo como del fin no lucrativo, aunque desde un punto de vista teórico comparta algunos atributos de ambos.

La "Rochdale Society of Equitable Pioneers" -que fue fundada el 24 de octubre de 1844 y abrió las puertas de su primer almacén el 21 de diciembre de ese mismo año en Rochdale, cerca de Manchester, en el Reino Unido- es considerada casi universalmente como la primera manifestación organizada de este tipo de organización empresarial al que incluso, hoy en día, nos venimos refiriendo con la denominación y esencia de "cooperativa"17.

La cooperativa de Rochdale empezó sus operaciones vendiendo alimentos básicos a sus socios y en el interés de los mismos. En la declaración de sus objetivos se enunciaba que la cooperativa operaba "para el beneficio pecuniario y el mejoramiento de las condiciones sociales y domésticas de sus socios" a través de varias actividades económicas, comenzando por "el establecimiento de un almacén para la venta de alimentos", e incluyendo la fabricación de productos para el empleo de personas desempleadas o subempleadas, así como la compra o la renta de propiedades de tierra para cultivar por parte de los socios.

Los objetivos de la Rochdale Society sustancialmente coinciden con los que el Derecho cooperativo vigente atribuye a las cooperativas. De hecho, si bien pueden encontrarse entre los ordenamientos jurídicos diferencias acerca de su extensión y naturaleza, es posible afirmar que las cooperativas están concebidas por la ley como entidades que conducen una empresa en el interés de sus socios como consumidores, proveedores o trabajadores de la misma empresa ${ }^{18}$.

\footnotetext{
${ }^{16}$ Con esto no se quiere decir que el fin de mutualidad caracteriza sólo a las cooperativas.

${ }^{17}$ Sin embargo, es comúnmente aceptado que antes de la constitución de la cooperativa de Rochdale, y no solo en el Reino Unido, ya existían otras entidades de tipo cooperativo. Rochdale se convirtió así en la precursora del cooperativismo moderno, principalmente debido a la adopción y formalización de normas de conducta específicas, que contribuyeron definitivamente a su éxito e inspiraron posteriormente al movimiento cooperativo y a la Alianza Cooperativa Internacional en la formulación de los valores y principios cooperativos. A este respecto las citas serían innumerables: basta mencionar aquí (Gide, 1921: 13 y ss.); (Fauquet, 1951: 57 y ss.); (Digby, 1948: 9 y ss.); y, más recientemente, entre otros, (Birchall, 2011: 6); (Sanchez Bajo y Roelants, 2011: $115)$.

${ }^{18}$ Véase (Cracogna, Fici y Henry, 2013), donde este punto está normalmente tratado en la tercera sección de los varios capítulos en su parte III.

Los principios de la ACI no son tan precisos en este sentido, si bien ellos hacen referencia al hecho de que la cooperativa actúa en el interés de sus socios (véase la definición), que son usuarios de los servicios proporcionados por la primera (primer principio), y hablan de "transacciones" de los miembros con la cooperativa (tercer principio).
}

REVESCO No 117 - MONOGRÁFICO: Las sociedades cooperativas construyen un mundo mejor - ISSN: 1885-8031 - www.ucm.es/info/revesco 
Este objetivo organizativo puede denominarse "fin mutualista" o "fin de mutualidad", aunque es verdad que sólo en algunos ordenamientos la ley o la doctrina jurídica adopten esta precisa fórmula para identificar el objetivo de las cooperativas y distinguirlo de los de otros entes jurídicos ${ }^{19}$.

Más concretamente, el fin de mutualidad consta de dos elementos: el objetivo final de beneficiar a los socios y el desarrollo de una concreta actividad para lograr este objetivo; es decir, una actividad de empresa con los socios en calidad de consumidores de los bienes o servicios proporcionados por la empresa (cooperativa de consumo), de proveedores de los bienes o servicios empleados por la cooperativa para desarrollar la empresa (cooperativa de producción), o de trabajadores de dicha impresa (cooperativa de trabajo) ${ }^{20}$.

Puesto que el objetivo final de beneficiar a los socios puede encontrarse también en otras entidades jurídicas, más concretamente, en todas las que persiguen un fin "interno", la especificidad del fin de mutualidad (y de las cooperativas que lo persiguen) reside en la concreta actividad, que es esencial para que la cooperativa consiga su objetivo final y para que sus socios satisfagan sus intereses detrás de la constitución de la cooperativa ${ }^{21}$. Oportunamente, entonces, algunas leyes cooperativas atribuyen una denominación específica a esta actividad y la someten a una disciplina particular ${ }^{22}$.

${ }^{19}$ En el Derecho cooperativo italiano la propia definición de cooperativa incluye el "fin mutualista" (scopo mutualistico) de este tipo de sociedad: véase el art. 2511 del Código civil italiano. Aunque la ley cooperativa estatal no utilice la expresión "mutualidad", ésta es común en la doctrina española: véase, por ejemplo, si bien críticamente (sosteniendo la crisis del principio de mutualidad), (Gadea et al, 2009: 37, 417 y ss.).

${ }^{20}$ Sobre la necesidad de identificar el fin de las cooperativas con referencia no solo al objetivo final de ellas, sino también a la manera en que este objetivo se persiga, véase (Digby, 1948: 7): "there is... something more precise which distinguishes co-operative from other business activities, something which belongs partly to end and partly to means". En la literatura no jurídica, la tripartición se encuentra, entre otros, en (Birchall, 2011: 3 y ss.).

${ }^{21}$ Esta actividad particular con los socios es una peculiaridad de la forma jurídica cooperativa que, correctamente entendida, contribuye significativamente a su distinción de las sociedades comerciales. En las sociedades comerciales, como en cualquier otra entidad con ánimo de lucro, la actividad económica es simplemente un medio para conseguir los objetivos finales del ente, y es irrelevante si esta actividad se desarrolla con los socios (ni, también si fuera este el caso, si los socios tienen derecho a un tratamiento mejor que los terceros no socios). Por el contrario, las cooperativas son establecidas y existen para conducir una empresa que pueda satisfacer directamente los intereses de sus socios-consumidores/proveedores/trabajadores. Por ello, entre otras razones, no parece convincente la tesis del profesor Hansmann según la cual las sociedades de capitales con ánimo de lucro no serían, en sustancia, tan diferentes de las cooperativas, puesto que son "cooperativas de capitales" (véase Hansmann, 1996: 11 y ss.); y, para una crítica de esta tesis, (Fici, 2013b: 25 y ss.).

${ }^{22}$ El ejemplo más fácil es el de la "actividad cooperativizada" en el Derecho cooperativo español. En América Latina la ley normalmente hace referencia a los "actos cooperativos", mientras que en el Derecho italiano se habla de "relaciones mutualistas": sobre este punto, véase recientemente (Bonfante, 2014: 136 y ss.).

REVESCO No 117 - MONOGRÁFICO: Las sociedades cooperativas construyen un mundo mejor - ISSN: 1885-8031 - www.ucm.es/info/revesco 


\section{3. ... Y SU COMPATIBILIDAD CON LA FUNCIÓN SOCIAL DE LAS COOPERATIVAS}

En la clasificación que hemos propuesto, el fin de la cooperativa es un fin “interno/egoísta”, ya que los socios se benefician de su actividad y resultados ${ }^{23}$. Por lo que se refiere a este aspecto, por lo tanto, las cooperativas no son diferentes de las sociedades lucrativas.

¿Cómo se explica, entonces, la función social de las cooperativas y su fomento por parte de los poderes públicos? ¿Cómo justificar su inclusión entre las entidades de la economía social? ¿No existe contradicción entre el fin de mutualidad y la función social? ¿Cómo se motiva la afirmación según la cual las cooperativas contribuyen a la construcción de un mundo mejor? ${ }^{24}$.

En efecto, la función social de las cooperativas, como veremos, deriva de varios elementos de su identidad jurídica, y no es incompatible con la persecución del fin de mutualidad, que, todo lo contrario, permite la realización de valores de relevancia constitucional relativos a la persona.

Teniendo un fin de mutualidad y no de lucro, las cooperativas no se proponen maximizar el rendimiento del capital invertido por los socios, sino satisfacer necesidades de diferente naturaleza, a través de una empresa, que, como hemos visto, se desarrolla con lo socios, y (lo que es aún más importante) en el interés de los mismos.

Los socios deciden establecer una cooperativa no ya porque quieren invertir sus capitales, sino porque quieren satisfacer necesidades propias por medio de una empresa que ellos puedan dirigir y controlar. En definitiva, para la satisfacción de sus necesidades concretas las personas no confían en el capital (de otros), sino se auto-organizan. El capital deja de ser necesario, o más bien, deja de ser el fin de la empresa y se convierte en un mero instrumento, un factor de producción entre los otros, al servicio de la persona.

\footnotetext{
${ }^{23}$ El fin mutualista requiere que estos resultados sean normalmente y preferentemente transferidos a los socios como "retornos cooperativos", es decir, en relación y en proporción a la actividad cooperativizada realizada con la cooperativa (y no al capital detentado).

${ }^{24}$ Así como se destacaba en el lema de las Naciones Unidas cuando proclamaron el 2012 año de las cooperativas.
}

REVESCO No 117 - MONOGRÁFICO: Las sociedades cooperativas construyen un mundo mejor - ISSN: 1885-8031 - www.ucm.es/info/revesco 
Además, las necesidades satisfechas por la cooperativa podrían tener naturaleza fundamental, en la medida en que pertenezcan a la esfera existencial de la persona, como la necesidad de trabajo o de vivienda. En este último caso la función social de las cooperativas aumenta de valor.

En breve, en razón de su fin de mutualidad, la cooperativa es una forma jurídica que permite y facilita la auto-organización y auto-gestión de las personas en la actividad económica. En cuanto tal, se funda en las personas y sus necesidades, y no en el capital. Su valor cual instrumento de desarrollo de la personalidad individual parece evidente, sobre todo si se relaciona con los otros aspectos de la identidad cooperativa que determinan su función social, en particular la estructura democrática ${ }^{25}$.

De hecho, entonces, si bien el fin de mutualidad es un fin "interno" como el fin de lucro, el primero es un fin sustancialmente diferente del segundo. El "egoísmo" de la cooperativa es un egoísmo particular y distinto del de las sociedades con ánimo de lucro, y por eso no es incompatible con la persecución de una función social, sino que la realiza ${ }^{26}$. Que sea así lo demuestran también las leyes cooperativas que otorgan un tratamiento fiscal específico y más favorable únicamente a las cooperativas que tengan y respeten un

\footnotetext{
${ }^{25}$ El Derecho cooperativo, como muchas veces ocurre (véase, entre otros, el art. 2514 del Código civil italiano; los artículos 14 y 15 de la Ley general francesa de cooperativas $n^{\circ} 47 / 1775$; y el art. 48 de la Ley estatal española $n^{\circ}$ 27/1999), debe proteger la mutualidad cooperativa limitando la cantidad de intereses que la cooperativa puede destinar a la remuneración del capital aportado por los socios, porque sin previsión de estos límites, el valor de la mutualidad desaparecería y se impondría el valor del lucro. Esto debe ser entendido como un caveat para todos los que puedan pensar que las cooperativas deberían ser libres de distribuir los beneficios como las sociedades lucrativas porque de otra manera serían perjudicadas en la competencia con estas. Sea como sea, sin embargo, el hecho es que, si fuera así, no habría competencia entre distintas formas jurídicas porque estas formas no serían distintas. La cooperativa sería homologada a la sociedad con ánimo de lucro. Esto significa que los problemas de la financiación de las cooperativas deben ser resueltos en manera diferente y compatible con la esencia de la cooperativa. Claramente, aquí se hace referencia a los intereses sobre el capital invertido (a la remuneración de las aportaciones al capital social) y no ya a los retornos cooperativos en sentido estricto, o sea, a las sumas asignadas a los socios (y originadas de su actividad cooperativizada con la cooperativa) en razón y en medida de su participación a la actividad cooperativizada (véase, por ejemplo, el art. 58, apartado 4, de la Ley estatal española ${ }^{\circ}$ 27/1999). La distribución de los retornos cooperativos realiza el fin mutualista (aunque ex post) y no se opone a éste.

${ }^{26}$ Sin embargo, una puntualización se hace aquí necesaria. Si la cooperativa está formada por entidades jurídicas no cooperativas y con ánimo de lucro (tal como es posible en algunos ordenamientos, como el italiano, que no ponen límites en este sentido), su fin de mutualidad no satisface intereses de carácter personal, sino que es un instrumento de lucratividad, lo que reduce o más bien anula la función social de la forma jurídica cooperativa. Asimismo, no todas las cooperativas tienen la misma función social, lo que depende de la naturaleza de las necesidades que esas satisfagan. Como se apuntó en el texto, una cooperativa que satisfaga necesidades básicas o de naturaleza fundamental de la persona, como las de trabajo o de vivienda, estaría en un nivel superior de una hipotética escala de valores sociales. Por lo tanto, a la hora de promover las cooperativas, el legislador debería tener en cuenta las diferentes clases de cooperativas (como por ejemplo ocurre en Italia donde, con referencia a la exención fiscal de los beneficios destinados a reservas indivisibles, el régimen varía en función del género y clase de la cooperativa: véase (Fici, 2013c: 497)).
}

REVESCO No 117 - MONOGRÁFICO: Las sociedades cooperativas construyen un mundo mejor - ISSN: 1885-8031 - www.ucm.es/info/revesco 
determinado mínimo de actividad cooperativizada con los socios (respecto a la actividad con terceros no socios) ${ }^{27}$.

Además, lo que refuerza la antedicha conclusión es que el fin (“egoísta”) de mutualidad es el principal pero no el único fin de las cooperativas, que destinan parte de los recursos que generan en beneficio de terceros no socios, manifestando así una cierta propensión al altruismo, su compromiso hacia "los demás".

Ya los principios de la ACI prevén asignaciones "externas" de recursos por parte de la cooperativa, cuando:

- incluyen no socios (más concretamente, dirigentes electos, gerentes y empleados) a los que la cooperativa brinda educación y formación (quinto principio);

- hacen referencia al deber de la cooperativa de informar al público en general, particularmente a jóvenes y creadores de opinión, acerca de la naturaleza y los beneficios del cooperativismo (quinto principio);

- obligan a las cooperativas a trabajar de manera conjunta para fortalecer el movimiento cooperativo (sexto principio);

- disponen que la cooperativa trabaje para el desarrollo sostenible de su comunidad (séptimo principio).

\footnotetext{
${ }^{27}$ El mejor ejemplo de esto lo ofrece el Derecho cooperativo italiano (sin embargo, una situación similar puede encontrarse en otros ordenamientos, por ejemplo, en Dinamarca, donde este mínimo es el 75\%: véase (Fjørtoft y Gjems-Onstad, 2013: 581); y en el Reino Unido, donde hay un específico tratamiento fiscal de las entidades, no sólo cooperativas, "enteramente mutuales": véase (Snaith, 2013: 753 y ss.). Este reconoce la distinción entre las cooperativas de mutualidad predominante (CMP) y las cooperativas de mutualidad no predominante u otras cooperativas (OC). Las CMP son cooperativas que operan de manera predominante con sus socios como usuarios de los bienes y servicios provistos por la cooperativa (en las cooperativas de consumo), como proveedores de los bienes y servicios utilizados por la cooperativa para su actividad económica (en las cooperativas de productores) o como trabajadores (en las cooperativas de trabajo) (art. 2512 del Código civil italiano). La condición de mutualidad predominante debe ser documentada de manera analítica destacando en el balance de la cooperativa que, dependiendo del tipo de "transacción mutual", (a) las ventas de los socios usuarios deben ser superiores al $50 \%$ de la totalidad de las ventas (en las cooperativas de consumo); (b) los costes laborales de los socios trabajadores deben ser superiores al 50\% del total de los costes laborales (en las cooperativas de trabajo); o (c) los costes de producción de los bienes y servicios prestados por los socios deben ser superiores al 50\% del total de los costes de producción (en las cooperativas de producción) (art. 2513 del Código civil italiano). En cambio, las OC no están obligadas a desarrollar una actividad predominante con sus socios ni a informar sobre el volumen de actividades realizadas con ellos. A diferencia de las OC, las CMP también están sujetas a las limitaciones sobre la distribución de beneficios en virtud del art. 2514 del Código civil italiano. La distinción entre estas dos tipologías de sociedad cooperativa no es muy relevante bajo el perfil del gobierno de la cooperativa, sino bajo el perfil fiscal, puesto que, en principio sólo las CMP son destinatarias de un tratamiento fiscal específico.
}

REVESCO N 117 - MONOGRÁFICO: Las sociedades cooperativas construyen un mundo mejor - ISSN: 1885-8031 - www.ucm.es/info/revesco 
En efecto, varias leyes cooperativas en el mundo aplican estos principios, obligando a las cooperativas a realizar asignaciones "externas" en favor de no socios (o futuros socios), otras cooperativas, el movimiento cooperativo, y la comunidad.

Entre las disposiciones legales más significativas en este sentido, cabe destacar las que obligan a las cooperativas a:

- constituir reservas indivisibles, también previendo que, en caso de disolución de la cooperativa, éstas sean destinadas a otras cooperativas o a asociaciones de representación del movimiento cooperativo u otra adjudicación desinteresada ${ }^{28}$;

- establecer fondos de educación y promoción cooperativa que no sean dirigidos solamente a los $\operatorname{socios}^{29}$

- destinar parte de sus beneficios a asociaciones de representación del movimiento cooperativo para que los usen para la promoción del cooperativismo (educación cooperativa, creación de nuevas cooperativas, etc.) ${ }^{30}$;

- destinar parte de sus beneficios a beneficencia o iniciativas en favor de la comunidad ${ }^{31}$.

\footnotetext{
${ }^{28}$ La reservas indivisibles fortalecen la cooperativa, pero también pueden ser vistas como una forma de altruismo hacia futuros cooperadores que al ingresar (lo que además está favorecido por el principio de la puerta abierta) pueden aprovechar las reservas acumuladas. Cuando estas reservas son irrepartibles entre los socios también en el caso de disolución de la cooperativa, su destino externo es evidente. En la legislación cooperativa en Europa hay ejemplos de leyes que prevén reservas indivisibles de naturaleza obligatoria pero repartibles entre los socios a la disolución de la cooperativa, y ejemplos de leyes que prevén su irrepartibilidad también en caso de disolución: para un marco comparativo véase (Fici, 2013a, p. 60 y ss.

${ }^{29}$ Esta es una manifestación de altruismo hacia terceros no socios y el movimiento cooperativo en general. Un ejemplo, por todos, lo ofrece el art. 56 de la Ley estatal española ${ }^{\circ} 27 / 1999$.

${ }^{30}$ Aquí la cooperativa es altruista hacia otros cooperadores y otras cooperativas. El ejemplo por excelencia lo ofrece el Derecho cooperativo italiano. Las cooperativas italianas están obligadas a destinar el 3\% del total de sus beneficios anuales a "fondos mutualistas para la promoción y el desarrollo del movimiento cooperativo" establecidos por las asociaciones nacionales de representación, asistencia y protección del movimiento cooperativo. Además, cuando una cooperativa italiana (más precisamente, una CMP) se disuelve o se transforma en otro tipo de sociedad, sus activos residuales, salvo el capital desembolsado y los dividendos devengados pero no distribuidos, son conferidos a los fondos mutualistas. Los fondos mutualistas tienen como único fin la promoción y financiación de nuevas empresas cooperativas y de iniciativas de desarrollo cooperativo. En este sentido, pueden promover el establecimiento de cooperativas y consorcios de cooperativas, así como poseer participaciones o acciones de cooperativas o de sus sociedades filiales. Asimismo, pueden financiar proyectos concretos de desarrollo de cooperativas y consorcios de cooperativas, organizar y gestionar la formación de los responsables del sector cooperativo, y promover estudios e investigaciones sobre cuestiones de carácter socioeconómico que sean de especial interés para el movimiento cooperativo. Sobre la importancia de esta regulación para la creación y fortalecimiento del movimiento cooperativo, véase (Fici, 2014b: 103 y ss.).

${ }^{31}$ Esto es lo que prevé, por ejemplo, la ley italiana con respecto a los bancos de crédito cooperativo: véase el art. 37, apartado 3, Decreto legislativo $\mathrm{n}^{\circ}$ 385/1993, y (Cusa, 2013: 111 y ss.).
}

REVESCO No 117 - MONOGRÁFICO: Las sociedades cooperativas construyen un mundo mejor - ISSN: 1885-8031 - www.ucm.es/info/revesco 


\section{LA FUNCIÓN SOCIAL DE LA ORGANIZACIÓN COOPERATIVA}

La función social de la cooperativa no deriva solamente de su fin mutualista, sino también (y no sería erróneo añadir, sobre todo) de su concreta organización interna o estructura de governance.

Esta estructura se articula alrededor de tres principios: el principio democrático; el principio de autogobierno, y el principio de la "puerta abierta". Estos principios se encuentran ya delineados en los principios de la ACI (véanse respectivamente el segundo, el cuarto y el primer principio) y en general las leyes cooperativas en el mundo los siguen y aplican ${ }^{32}$.

El principio democrático implica que en una cooperativa cada socio tiene un voto independientemente del capital aportado ${ }^{33}$. Por lo tanto, la causa y medida de la participación no es el capital, como en las sociedades de capitales, sino la persona en cuanto tal e independientemente del capital que posea.

Autogobierno cooperativo significa que las cooperativas son sociedades controladas por sus socios, no solamente en sentido formal, lo que se sería válido para todas las sociedades, sino también en sentido sustancial. Esto es también un efecto del principio democrático. A diferencia de las sociedades lucrativas, las cooperativas no pueden ser sujetas a dirección y control externos, ni pueden ser dirigidas y controladas por un solo socio o una minoría de $\operatorname{socios}^{34}$. Este principio incentiva la participación efectiva de los socios en la vida social, a la que puede contribuir también la ley a través de disposiciones de distinto tipo ${ }^{35}$.

Una governance societaria de este tipo tiene una función social porque se basa en la centralidad de la persona, en su prevalencia sobre el capital, y promueve su participación en la vida económica. Utilizando las palabras de la Constitución italiana (art. 2) da lugar a una

\footnotetext{
${ }^{32}$ Para un panorama comparativo, véase limitado a Europa (Fici, 2013a: 61 y ss.); y más ampliamente los varios capítulos en la parte III de (Cracogna et al, 2013).

${ }^{33}$ En la legislación cooperativa se encuentran varias excepciones. Sin embargo, cualquiera que sea esta excepción, la ley impide el control por un sólo socio o clase de socios, limitando el número de votos que un socio (o clase de socios) puede ejercer en cada asamblea. Para referencias, véase la literatura citada en la precedente nota a pie de página. Cabe subrayar que en los principios de la ACI, la regla "un socio, un voto" se refiere solamente a las cooperativas de primer nivel (entre personas físicas), mientras que por lo que se refiere a las de nivel superior se establece: "las cooperativas de otros niveles también se organizan con procedimientos democráticos".

${ }^{34}$ En este sentido, el cuarto principio de la ACI aclara que las cooperativas, "si entran en acuerdos con otras organizaciones (incluyendo gobiernos) o tienen capital de fuentes externas, lo realizan en términos que aseguren el control democrático por parte de sus miembros y mantengan la autonomía de la cooperativa”.

${ }^{35}$ Por ejemplo, autorizando la subdivisión de la asamblea de los socios en asambleas territoriales; o la exclusión de la cooperativa de los socios que no toman parte en la asamblea; etc.
}

REVESCO N 117 - MONOGRÁFICO: Las sociedades cooperativas construyen un mundo mejor - ISSN: 1885-8031 - www.ucm.es/info/revesco 
formación social que favorece el desarrollo de la personalidad del individuo. Una forma jurídica de empresa regida por estos principios constituye también -haciendo nuevamente referencia a la Constitución italiana (art. 3, apartado 2) - un instrumento de remoción de aquellos obstáculos de naturaleza económica o social que de hecho impiden la participación efectiva de los ciudadanos más débiles en la vida económica y política del país ${ }^{36}$.

En otras palabras, en virtud de sus específicos principios de governance, las cooperativas son instrumentos de democracia económica y de igualdad sustancial. Permiten a todos, también a los sin capital, tomar parte en la vida económica y social, y, en tal manera, constituyen también "escuelas de empresariado"37.

Otro aspecto de la governance cooperativa que contribuye a su función social, sobre todo puesto en relación con los perfiles económicos que hemos analizado antes (más concretamente, con la existencia de reservas indivisibles), es la apertura de la afiliación (o membresía) a terceros, según lo previsto por el primer principio de la ACI ("Las cooperativas son organizaciones voluntarias abiertas para todas aquellas personas dispuestas a utilizar sus servicios y dispuestas a aceptar las responsabilidades que conlleva la membresía sin discriminación de género, raza, clase social, posición política o religiosa”) y facilitado por las leyes cooperativas, que normalmente prevén que las cooperativas son sociedades con capital y número de socios variables ${ }^{38}$.

Si bien este principio no otorga a los terceros el derecho subjetivo absoluto de ingresar, sino solamente les reconoce un interés que las leyes cooperativas protegen de diferente manera ${ }^{39}$, éste manifiesta un diferente aspecto de la función social de la cooperativa, porque la admisión de nuevos socios constituye una modalidad de compartir los beneficios de una empresa con terceros, y por lo tanto una forma de altruismo (de la cooperativa y de sus socios actuales). En este sentido, paradójicamente, las cooperativas son sociedades "abiertas", mientras que las sociedades de capital, incluso las cotizadas, son sociedades "cerradas". Esto porque en las sociedades de capitales los titulares de las acciones pueden cambiar con

\footnotetext{
${ }^{36}$ Véase (Nigro, 1980: 18 y ss.).

${ }^{37}$ Como la Comisión europea ha subrayado en su Comunicación de 2004 sobre la promoción de las sociedades cooperativas en Europa.

${ }^{38}$ Véase, por ejemplo, el art. 1, apartado 2, del Reglamento ${ }^{\circ}$ 1435/2003 de la Sociedad cooperativa europea (SCE). Capital variable significa que su variación por el ingreso de nuevos socios no requiere una modificación de los estatutos sociales ni otras formalidades.

${ }^{39}$ Sobre la interpretación y aplicación de este principio por la ley, véase (Fici, 2013a: 52-53, 62-63).
}

REVESCO N 117 - MONOGRÁFICO: Las sociedades cooperativas construyen un mundo mejor - ISSN: 1885-8031 - www.ucm.es/info/revesco 
facilidad, pero no cambia con la misma facilidad el número total de las acciones, y consecuentemente el número total de los beneficiarios ${ }^{40}$.

\section{LAS COOPERATIVAS DE INTERÉS GENERAL Y EL PROBLEMA DE LA REGULACIÓN DE LA EMPRESA SOCIAL}

A pesar de las destacadas asignaciones externas, las cooperativas permanecen orientadas hacia sus socios, cuyos intereses aspiran (principalmente) a satisfacer. Las cooperativas son entidades mutualistas y el de mutualidad es un fin "interno". Los socios de una cooperativa, por lo tanto, son "propietarios" en el sentido de Hansmann, puesto que detentan tanto el derecho de controlar la empresa como el derecho de apropiarse de los beneficios de ésta (si bien típicamente y principalmente en forma de retornos) ${ }^{41}$. En consecuencia, las cooperativas no pueden ser consideradas entidades sin ánimo de lucro, dado que sus beneficiarios son las propias personas que las controlan ${ }^{42}$. Tienen una función social, pero no son entidades (principalmente) altruistas o solidarias ${ }^{43}$.

Sin embargo, esta última conclusión es valida sólo por lo que se refiere a aquel (más tradicional) tipo de cooperativa que corresponde con el modelo de cooperativa de Rochdale y al que se refiere la ACI en sus principios, es decir, la cooperativa mutualista que los socios establecen para satisfacer sus propias necesidades (diferentes de la de inversión del capital). En cambio, la antedicha conclusión no sería valida con referencia a otro adicional (y más reciente) modelo de cooperativa, que los legisladores en el mundo -comenzando por el legislador italiano a través de la Ley $n^{\circ} 381 / 1991$ sobre las cooperativas sociales ${ }^{44}$ - están introduciendo cada vez más en sus ordenamientos jurídicos.

\footnotetext{
${ }^{40}$ Véase (Fici, 2013a: 50 y ss.).

${ }^{41}$ Según (Hansmann, 1996: 11 y ss., 17 y ss.), los propietarios de una empresa "son aquellas personas que comparten dos derechos formales: el derecho de controlar la empresa y el derecho de apropiarse de los beneficios de la empresa, es decir, de las ganancias residuales"; por eso, los socios de una entidad non profit no pueden considerarse "propietarios", porque les falta el derecho de apropiarse de los beneficios residuales de la empresa, que, junto al derecho de controlar la empresa, es un elemento esencial del concepto de propiedad de la empresa.

${ }^{42}$ Esto no significa que no sean diferentes de las sociedades de capitales, porque -además de la función social que puede adscribirse a la cooperativa (y no a la sociedad de capitales) en virtud de las antedichas características- sólo en una cooperativa los socios (propietarios de la empresa) son los directos usuarios de la empresa, lo que no ocurre en las sociedades de capitales. He tratado este punto, en oposición a la tesis del profesor Hansmann en (Hansmann, 1996), en (Fici, 2013b: 25 y ss.).

${ }^{43}$ Esto no significa que no puedan estar incluidas en una única categoría, como entidades de la economía social.

${ }^{44}$ Por lo menos, por lo que se refiere a Europa y por lo que el autor de este artículo sepa. Sin embargo, es verdad que la "community benefit society", introducida en el Reino Unido por el Industrial and Provident Societies Act de 1965, tiene una historia legislativa más larga que la cooperativa social italiana de 1991. Sin embargo, la
}

REVESCO No 117 - MONOGRÁFICO: Las sociedades cooperativas construyen un mundo mejor - ISSN: 1885-8031 - www.ucm.es/info/revesco 
Las cooperativas sociales italianas -como las cooperativas francesas de interés colectivo, las cooperativas españolas de iniciativa social y las cooperativas portuguesas de solidaridad social, para mencionar sólo algunas ${ }^{45}$ - "persiguen el interés general de la comunidad" 46 y no el interés de sus socios. No son cooperativas mutualistas (con fin “interno/egoísta” de mutualidad), sino cooperativas de interés general (por lo tanto, con un fin externo/altruista $)^{47}$.

Los estudiosos del Derecho cooperativo, entonces, tienen que tomar conocimiento del hecho que hoy no hay solamente cooperativas mutualistas, que existe una tendencia legislativa a la introducción de cooperativas sin fin de mutualidad y con un fin de interés colectivo o general, que la ley tiende a separar las cooperativas de la persecución de un fin interno de mutualidad.

Por consiguiente, la ciencia jurídica cooperativa debería dedicar especial atención a este nuevo modelo de cooperativa, que debido a su diferente objetivo, presenta específicos problemas de regulación ${ }^{48}$.

El concepto de cooperativa social (o de interés colectivo, de iniciativa social, de solidaridad social, cualquiera que sea su denominación) nos lleva a un problema legislativo de gran interés actual, que es el de la regulación de la "empresa social".

La empresa social es una figura de la cual todavía no existe una noción uniforme y compartida a nivel europeo ${ }^{49}$. Mediante ella nosotros entendemos una entidad privada que

"community benefit society" no tiene necesariamente que tener una estructura cooperativa y operar en base al principio "un socio, un voto", y en la ley está concebida como una alternativa a la cooperativa (en el sentido que una sociedad puede registrarse o como sociedad cooperativa de buena fe o como una community benefit society). ${ }^{45}$ Véanse respectivamente el art. 19 quinquies y ss. de la Ley n ${ }^{\circ} 47-1775$; el art. 106 de la Ley n ${ }^{\circ} 27 / 1999$; el Decreto-Ley $n^{\circ} 7 / 1998$. Las cooperativas sociales existen también, entre otros, en Grecia, Hungría y Polonia, y fuera de Europa, en Corea del Sur.

${ }^{46}$ Así prevé expresamente la Ley italiana n 381/1991 al identificar el fin de las cooperativas sociales.

${ }^{4}$ Sin embargo, es verdad que esta conclusión podría mutar parcialmente según el ordenamiento jurídico considerado, porque la percepción del concepto de cooperativa social es variable. En algunos ordenamientos, como el italiano, parece bastante claro que las cooperativas sociales puedan perseguir únicamente un fin de interés general y no puedan operar en favor de sus socios en cuanto tales (aunque hay autores que mantienen que la cooperativa social también es mutualista: acerca de este debate véase (Fici, 2004). En cambio, en otros ordenamientos, como el español, las cooperativas sociales (o de iniciativa social, como se denominan allí) no son concebidas en oposición a las cooperativas mutualistas, sino como un tipo específico de éstas.

${ }^{48}$ Por ejemplo, la estructura de gobierno de una cooperativa de interés general debería ser diseñada por la ley en congruencia con su objetivo particular, que tiene naturaleza externa y no interna (como en una cooperativa mutualista). Esto podría implicar dar expresión a beneficiarios que no son socios o, más en general, a representantes de la comunidad en la que la cooperativa opera.

${ }^{49}$ Sin embargo, véase Comisión Europea, COM (2011) 682 final, de 25.10.2011, Iniciativa en favor del emprendimiento social, pp. 2 y ss.: "Por 'empresa social', la Comisión entiende las empresas cuyo objetivo

REVESCO No 117 - MONOGRÁFICO: Las sociedades cooperativas construyen un mundo mejor - ISSN: 1885-8031 - www.ucm.es/info/revesco 
desarrolla, sin ánimo de lucro y para finalidades de interés común o general, una actividad económica de utilidad social o de interés comunitario ${ }^{50}$.

Si ésta es la noción adoptada, no cabe duda de que las cooperativas sociales entran en esta categoría; constituyen una forma jurídica de empresa social; y lo que es más relevante, son empresas sociales controladas de forma democrática por sus socios.

En efecto, la cooperativa social ha sido la primera forma de empresa social reconocida por la ley. Más precisamente, el legislador ha considerado la forma cooperativa la más apropiada para la regulación de la empresa social, esto es, ha empleado la forma jurídica cooperativa como marco jurídico específico para la empresa social. Por lo tanto, jurídicamente, la empresa social de primera generación deriva de una adaptación del tipo legal de la cooperativa, y de verdad esto no sorprende, teniendo ya la forma jurídica cooperativa una función social, como hemos intentado demostrar en estas páginas.

Sin embargo, el punto es que, en la legislación más reciente en temas de empresa social, el legislador ha adoptado un enfoque diverso ${ }^{51}$.

De hecho, en algunos ordenamientos, la empresa social se presenta en la ley como un tipo particular de sociedad de capital que desarrolla una actividad de utilidad social (o de interés comunitario) con lucro limitado. En este caso, el marco jurídico para la empresa social lo ofrece el Derecho de las sociedades de capitales.

En Europa, el ejemplo más evidente sería la sociedad de interés comunitario (community interest company), contemplada en la Ley inglesa de sociedades de 2004 y

social de interés común es la razón de ser de la acción comercial, que se traduce a menudo en un alto nivel de innovación social; cuyos beneficios se reinvierten principalmente en la realización de este objetivo social; y cuyo modo de organización o régimen de propiedad, basados en principios democráticos o participativos u orientados a la justicia social, son reflejo de su misión". Entre los investigadores, también es muy conocida la definición que de ella ofrece la red EMES, o más bien los indicadores que EMES ofrece para calificar una entidad como empresa social: véase (Defourny y Nyssens, 2012: 7 y ss.).

${ }^{50}$ Véase (Fici, 2009: 77 y ss.).

${ }^{51}$ Sobre este punto, véase (Fici, 2015). Véase también (Priede Bergamini, López-Cózar Navarro y Rodríguez López, 2014).

REVESCO No 117 - MONOGRÁFICO: Las sociedades cooperativas construyen un mundo mejor - ISSN: 1885-8031 - www.ucm.es/info/revesco 
regulada por un específico reglamento de $2005^{52}$. El modelo societario es también lo que está siguiéndose en muchos Estados de los Estados Unidos de América ${ }^{53}$.

En otros ordenamientos, en cambio, la ley considera la empresa social como una especial categoría jurídica de organizaciones en la que pueden ser incluidas, independientiemente de su forma jurídica, todas las entidades que desarrollen sin ánimo de lucro una actividad de utilidad social. Esto permite constituir una empresa social no sólo en la forma de cooperativa, de asociación o de fundación, sino también en la forma de sociedad mercantil, siempre que esta sociedad posea los requisitos para adquirir la calificación de empresa social. El ejemplo más relevante de este tipo de legislación sobre la empresa social lo ofrece una ley italiana de $2006^{54}$.

La posibilidad de constituir una empresa social en forma de sociedad de capitales (aunque sin ánimo de lucro, como en Italia, o con lucro limitado, como en el Reino Unido) plantea delicadas cuestiones conexas con la oportunidad que la ley legitime empresas sociales no democráticas, dirigidas por el capital en lugar de las personas de los socios y por lo tanto controlable por parte de una sola persona (o grupos de personas) ${ }^{55}$, porque este es el efecto fundamental de la utilización del (o de la posibilidad de recurrir también al) Derecho de las sociedades de capitales como marco jurídico para la empresa social ${ }^{56}$.

La empresa social en forma de sociedad de capitales, entonces, es una organización cuya identidad como empresa social o como entidad de la economía social es más débil, si se considera la posible falta de democracia interna. El riesgo que la empresa social en forma de sociedad de capital sea empleada para estrategias de puro marketing parece alto. Alto parece también el riesgo de ofuscación de la diferencia entre el concepto de empresa social y el de

\footnotetext{
${ }^{52}$ Informaciones sobre este tipo de sociedad pueden encontrarse en el sitio web de la autoridad de registro y control: https://www.gov.uk/government/organisations/office-of-the-regulator-of-community-interestcompanies.

${ }^{53}$ Para una visión panorámica de esta legislación, también en relación a la legislación europea, véase (Esposito, 2013).

${ }^{54}$ Más concretamente el Decreto legislativo n 155/2006. Para un análisis, véase (Fici y Galletti, 2007); (Fici, 2007). Este modelo también caracteriza la Ley finlandesa de 2003 sobre la empresa social, entre otras.

${ }^{55}$ Si no hay limites a la participación de cada socio en el capital social o al número de los votos a que cada socio tenga derecho en una asamblea, como ocurre en el Derecho belga, donde este limite es 1/10 (véase art. 661, apartado, $1, \mathrm{n}^{\circ}$ 4, del Código belga de sociedades, con referencia a las sociedades con finalidad social).

${ }^{56}$ En un interesante artículo publicado en una revista norteamericana, un abogado inglés explica como ésta, la posibilidad de control, constituye la razón principal por la que la sociedad de interés comunitario (community interest company) ha sido introducida en el ordenamiento inglés, puesto que el Derecho de las organizaciones de caridad (charities) no permite que éstas sean controladas por una sola persona, incluso el fundador: véase (Lloyd, 2010: 31 y ss.).
}

REVESCO No 117 - MONOGRÁFICO: Las sociedades cooperativas construyen un mundo mejor - ISSN: 1885-8031 - www.ucm.es/info/revesco 
responsabilidad social empresarial, así como de una dañina invasión del sector de la economía social por parte de los actores de la economía capitalista y de sus lógicas específicas.

Todo esto requiere que la supremacía de la forma cooperativa para la regulación del fenómeno de la empresa social sea reafirmada. También por este motivo, un análisis de la función social de la forma jurídica cooperativa se presenta de gran actualidad, porque permite reflexionar sobre el valor añadido que el revestimiento jurídico de cooperativa atribuye a la empresa social, en virtud de la función social que la forma jurídica cooperativa ya contiene ${ }^{57}$.

\section{CONCLUSIONES}

Como bien destaca el profesor Dante Cracogna, "la dimensión social de las cooperativas surge de su propia naturaleza, a la cual la legislación y las regulaciones administrativas deben plegarse adecuadamente a fin de permitir el pleno desarrollo de todo su potencial" ${ }^{, 58}$.

De acuerdo con ello, este artículo ha reseñado brevemente los aspectos de la identidad jurídica de la cooperativa de que depende su función social. La función social de las cooperativas es el resultado de un fin y de una estructura organizativa típicos, impregnados de valores como mutualidad (en el sentido de autoayuda), altruismo y solidaridad, supremacía de la persona sobre el capital, democracia, participación y autogestión ${ }^{59}$.

La función social de la cooperativa se necesita puntualizar, depende de leyes que atribuyan a las cooperativas una específica identidad y la preserven. Sólo así es posible afirmar que las cooperativas son diferentes de los restantes tipos de sociedades y merecen un tratamiento particular coherente con sus rasgos típicos y capaz de premiar su contribución a la comunidad. De lo contrario, el fomento estatal de las cooperativas sería difícilmente justificable a la luz del Derecho de la competencia.

La función social de la cooperativa la convierte en el tipo más adecuado para dar forma jurídica al fenómeno de la empresa social, como lo demuestran las leyes de primera generación sobre la empresa social, que de hecho eran leyes de cooperativas sociales.

\footnotetext{
${ }^{57}$ Esto significa que, si se admite que una empresa social pueda ser constituida en cualquier forma jurídica, sería por lo menos necesario reconocer un tratamiento particular, por ejemplo bajo el Derecho fiscal, a las empresas sociales que tengan la forma de cooperativa.

${ }^{58}$ Véase (Cracogna, 2014).

${ }^{59}$ Sobre algunos de estos valores hemos reflexionado también en (Fici, 2014a).
}

REVESCO No 117 - MONOGRÁFICO: Las sociedades cooperativas construyen un mundo mejor - ISSN: 1885-8031 - www.ucm.es/info/revesco 
Estas leyes han introducido una distinción entre las cooperativas mutualistas (el modelo más tradicional) y las cooperativas de interés general, que la doctrina jurídica debe tomar en consideración, como está tratando de hacer el Grupo de estudio sobre el Derecho cooperativo europeo en su proyecto de redacción de los Principios de Derecho cooperativo europeo $^{60}$.

La reflexión sobre la función social de la forma jurídica cooperativa es esencial también a fin de guiar al legislador en la confección de un marco jurídico adecuado para el fenómeno de la empresa social, así parando o por lo menos atenuando una tendencia legislativa a implantar la empresa social en el cuerpo del Derecho de las sociedades mercantiles en lugar de el de las sociedades cooperativas, que sería el más natural para ella.

\section{BIBLIOGRAFÍA}

APARÍCIO MEIRA, D. (2013) A lei de bases de economia social portuguesa: do projeto ao texto final. CIRIEC-España, Revista Jurídica de Economía Social y Cooperativa, $\mathrm{n}^{\circ} 24$, pp. 21 y ss.

BIRCHALL, J. (2011) People-Centred Businesses. Co-operatives, Mutuals and the Idea of Membership. London: Palgrave MacMillan.

BONFANTE, G. (2014) La società cooperativa. Padova: Cedam.

CRACOGNA, D. (2014) Las cooperativas y su dimensión social [feche de consulta: 1 de diciembre de 2014]. Disponible en: http://www.derecho.uba.ar/publicaciones/pensar-enderecho/revistas/3/las-cooperativas-y-su-dimension-social.pdf.

CRACOGNA, D., FICI, A., HENRY, H. (2013) International handbook of cooperative law. Heidelberg: Springer

CUSA, E. (2013) Il diritto delle banche di credito cooperativo tra legge e contratto. Torino: Giappichelli.

DEFOURNY, J., y NYSSENS, M. (2012) El enfoque EMES de empresa social desde una perspectiva comparada. CIRIEC-España. Revista de Economía Pública, Social y Cooperativa, $\mathrm{n}^{\circ} 75$, pp. 7 y ss.

\footnotetext{
${ }^{60}$ De hecho, en la primera versión de estos Principios aparece la distinción entre "cooperativas mutualistas" y "cooperativas de interés general", que se basa en el fin predominante, es decir, realizar actividad cooperativizada con los socios en el interés de los mismos y operar en el interés de la comunidad, respectivamente: véase (Fajardo, Fici, Henrÿ, Hiez, Meira, Münkner y Snaith, 2013).
}

REVESCO No 117 - MONOGRÁFICO: Las sociedades cooperativas construyen un mundo mejor - ISSN: 1885-8031 - www.ucm.es/info/revesco 
DIGBY, M. (1948) The World Co-operative Movement. London: Hutchinson's University Library.

ESPOSITO, R.T. (2013) The social enterprise revolution in corporate law: a primer on emerging corporate entities in Europe and the United States and the case for the benefit corporation. William \& Mary business law review, $\mathrm{n}^{\circ} 4$, pp. 639 y ss.

FAJARDO, G., FICI, A., HENRŸ, H., HIEZ, D., MEIRA, D., MÜNKNER, H. H., y SNAITH I. (2013) El Nuevo grupo de estudio en Derecho cooperativo europeo y el proyecto "los principios del Derecho cooperativo europeo". CIRIEC-España. Revista Jurídica de Economía Social y Cooperativa, n² 24, pp. 331 y ss.

FAUQUET, G. (1951) The Co-operative Sector [traducción de la cuarta edición francesa publicada en 1942]. Manchester: Co-operative Union Limited.

FICI, A. (2004) Cooperative sociali e riforma del diritto societario. Rivista di diritto privato, pp. 75 y ss.

FICI, A. (2007) Impresa sociale. Digesto delle discipline privatistiche, sezione civile, aggiornamento, vol. 3, t. II. Torino: Utet.

FICI, A. (2009) Cooperatives and social enterprises: comparative and legal profiles. En: ROELANTS, B., Cooperatives and social enterprises. Governance and normative frameworks. Brussels: CECOP.

FICI, A. (2013a) Cooperative identity and the law. European Business Law Review, ${ }^{\circ}$ 24, p. 60 y ss.

FICI, A. (2013b) An introduction to cooperative law. En: CRACOGNA, D., FICI, A., HENRY, H., International handbook of cooperative law. Heidelberg: Springer.

FICI, A. (2013c) Italy. En: CRACOGNA, D., FICI, A., HENRY, H., International handbook of cooperative law. Heidelberg: Springer.

FICI, A. (2014a) Valores cooperativos, derecho cooperativo y jovenes. Deusto Estudios Cooperativos, $\mathrm{n}^{\circ} 4$, pp. 83 y ss.

FICI, A. (2014b) La cooperación entre cooperativas en el derecho italiano y comparado. Boletín de la Asociación Internacional de Derecho Cooperativo, $\mathrm{n}^{\circ}$ 48, pp. 103 y ss.

FICI, A. (2014c) La sociedad cooperativa europea: cuestiones y perspectivas, en CIRIECEspaña. Revista Jurídica de Economía Social y Cooperativa, (de próxima publicación).

FICI, A. (2015) Función y modelos de regulación de la empresa social (de próxima publicación).

REVESCO N 117 - MONOGRÁFICO: Las sociedades cooperativas construyen un mundo mejor - ISSN: 1885-8031 - www.ucm.es/info/revesco 
FICI, A., y GALLETTI, D. (2007) Commentario al decreto sull'impresa sociale. D. Lgs. 24 marzo 2006, n. 155. Torino: Giappichelli.

FJØRTOFT, T., y GJEMS-ONSTAD, O. (2013) Norway and scandinavian countries. En: CRACOGNA, D., FICI, A., HENRY, H., International handbook of cooperative law. Heidelberg: Springer.

GADEA, E., SACRISTÁN, F. Y VARGAS VASSEROT, C. (2009) Régimen jurídico de la sociedad cooperativa del siglo XXI. Madrid: Dykinson.

GIDE, C. (1921) Consumers' Co-operative Societies, [traducción de la tercera edición francesa publicada en 1917]. Manchester: Co-operative Union Limited.

HANSMANN, H. (1996) The ownership of enterprise. Cambridge-London: HUP.

LLOYD, S. (2010) Transcript. Creating the CIC. Vermont law review, ${ }^{\circ} 35$, pp. 31 y ss.

NIGRO, A. (1980) Sub art. 45. En G. BRANCA, Commentario della Costituzione, III, Bologna-Roma: Zanichelli-Foro italiano.

PRIEDE BERGAMINI, T., LÓPEZ-CÓZAR NAVARRO, C., y RODRÍGUEZ LÓPEZ, A. (2014) Análisis del marco económico-jurídico específico para los emprendedores sociales: un estudio comparado entre diversos países. CIRIEC-España. Revista de Economía Pública, Social y Cooperativa, $\mathrm{n}^{\circ} 80, \mathrm{pp} .5$ y ss.

SANCHEZ BAJO, C., y ROELANTS, B. (2011) Capital and the Debt Trap. Learning from Cooperatives in the Global Crisis. Basingstoke: Palgrave MacMillan.

SANTINI, G. (1973) Tramonto dello scopo lucrativo nelle società di capitali, en Rivista .di diritto civile, I, pp. 151 y ss.

SNAITH, I. (2013) United Kingdom. En: CRACOGNA, D., FICI, A., HENRY, H., International handbook of cooperative law. Heidelberg: Springer.

SPADA, P. (1974) La tipicità delle società. Padova: Cedam.

TORRES MORAlES, C. (2013) Peru. En: CRACOGNA, D., FICI, A., HENRY, H., International handbook of cooperative law. Heidelberg: Springer.

REVESCO No 117 - MONOGRÁFICO: Las sociedades cooperativas construyen un mundo mejor - ISSN: 1885-8031 - www.ucm.es/info/revesco 